\title{
Denkmöglichkeiten - Der 38. Deutsche Rechtshistorikertag in Münster
}

\author{
Miloš Vec*
}

Mit einem Fauxpas begann der Münsteraner Rechtshistorikertag. Er müsse beichten, erklärte der dritte Eröffnungsredner, dass er jene Konvention, die die Vorrednerin als selbstverständlich vorausgesetzt hätte, nicht gekannt habe und sie nun verletzen werde. Die zitierte Vorrednerin war Ursula Nelles, ihres Zeichens Juristin und Rektorin der Universität, und sie hatte eingangs erklärt, die Regel laute: „bei Grußworten niemals zur Sache sprechen " und auch sie werde heute, dieser rhetorischen Konvention folgend, selbstverständlich nichts zum Kongressgegenstand sagen. Tatsächlich hielt sie sich freundlich lächelnd an die Ankündigung, sprach ebenso wie der Dekan viel über die Münsteraner Universität und ihre Juristen. Ursula Nelles und HansMichael Wolffgang trugen mit Verve und Münsteraner Selbstbewusstsein vor, was ihnen wichtig schien: Die gute Stellung ihrer Fakultät im nationalen Vergleich, die Verknüpfungen der Juristen mit dem Exzellenzcluster und die Vorreiterstellung der Rechtshistoriker wurden mit Stolz herausgekehrt. Sie redeten nicht als Forscher und Lehrer, sondern als Spitzen einer Universitätsadministration, der ihre Repräsentations- und Marketing-Aufgabe selbstverständlich sind, gleich welchen Kongress man eröffnet, Bienen- oder Batterieforscher.

Das konnte man sicher so machen und es war auch nicht besonders ungewöhnlich. Dennoch irritierte dieses Schweigen den einen oder anderen Rechtshistoriker. Wie nun, wenn es geradezu ungewollt das Verhältnis von Geltendrechtlern zur Geschichte des Rechts abbilden würde? Wenn sich darin das manches Mal zu beobachtende wohlwollende Desinteresse auch von besonders exponierten Fachvertretern niederschlagen würde? Das wäre unter allen Aspekten bedrückend, und nicht zuletzt deswegen, weil die Rechtshistoriker immer wieder die verbindenden Aspekte zum geltenden Recht und seinen Vertretern als Legitimation evozieren. Ist das Lob der Grundlagenfächer wohlfeile Rhetorik, während sie in der Sache immer mehr an die Peripherie gedrängt werden und jenseits des Spezialistentums Unkenntnis historischer Zusammenhänge um sich greift? Die Frage, was die Fakultätskollegen auch inhaltlich mit jenem Fach, das sie schmückt, anfangen können, blieb jedenfalls bei diesen beiden offen.

Anders der dritte Mann, Hochschulrat Jürgen Kaube, Redakteur im Feuilleton der Frankfurter Allgemeinen Zeitung, der nichts von dieser Konvention wusste. Kaube

* PD Dr. Miloš Vec ist Privatdozent für Neuere Rechtsgeschichte, Rechtsphilosophie, Rechtstheorie und Zivilrecht an der Johann Wolfgang Goethe-Universität in Frankfurt am Main und leitet derzeit im Rahmen des Exzellenzclusters 243 „Formation of Normative Orders“ das Forschungsprojekt „Das Völkerrecht und seine Wissenschaft - 1789-1914“ am Max-Planck-Institut für europäische Rechtsgeschichte. Daneben wirkt er als Co-Direktor einer Projektgruppe „Paradoxes of Peace“ an der Universität Helsinki. 
witzelte zunächst auch, und zwar zunächst gegen seine Denomination, da er fand, dass „Hochschulrat kein Titel und keine Anrede“ sei. Dann aber wechselte er die Tonlage und stellte in seinem Grußwort einige tastende Überlegungen zur Rechtsgeschichte an, die ihm, obwohl er weder Historiker noch Jurist ist, immer besonders attraktiv vorgekommen sei. Kaube ließ die Zuhörer an einem Blick in sein Bücherregal teilhaben, referierte und kommentierte die dort vorhandenen rechtsgeschichtlichen Titel. Der zentrale Begriff, der in seiner Rede mehrmals aufblitzte, war so ungewöhnlich nicht, aber er würde in den nächsten Tagen gelegentlich zitiert werden. Kaube sprach von „Denkmöglichkeiten“, die die Rechtsgeschichte eröffnen würde und er hielt diese für wichtig. Was wäre ein Jurist, der nicht mit Bodin, Hobbes und Savigny bekanntgemacht worden wäre? Einem Juristen, der nur das geltende Recht kenne, würde eine Dimension fehlen. Den Rechtshistorikern attestierte Kaube die große Komplexität der Materie, die sich aber gerade von derjenigen der Historiker unterscheide. Kaube meinte, bei den Rechtshistorikern ein „normatives Element“ ausmachen zu können, das ihren Aussagen innewohne. Diese Überlegungen konnten - so viel Konvention und Normbefolgung verstand sich beim Grußwort des Hochschulrats dann doch von selbst - nicht im Anschluss diskutiert werden. Die Adresse an das Selbstverständnis der Rechtshistoriker, verbunden mit etwas theoretischer Reflexion, hatte jedenfalls die Legitimationsfrage angedeutet und eine mögliche Antwort gegeben.

Die Organisatoren des Rechtshistorikertags in Münster, Nils Jansen, Peter Oestmann und Reiner Schulze, hatten einige Reformen gewagt und alle sind sie ihnen gelungen. Das Programm wurde erkennbar gestrafft, statt neun Sektionen wie noch in Passau ${ }^{1}$ gab es diesmal nur vier, die wissenschaftliche Veranstaltung dauerte vom 15. bis 17. September und war damit insgesamt um einen ganzen Tag kürzer als 2008. Auch die Trennung in Germanistische, Romanistische und Kanonistische Sektionen wurde aufgegeben. Tagungsgebühren waren keine zu entrichten. Bei den Rednern wurde sanft auf Verjüngung gesetzt, da mancher das Durchschnittsalter bei den Plenarvorträgen in Passau mit 65,8 Jahren doch als zu hoch empfunden hatte. ${ }^{2}$ Tatsächlich änderte sich das Gesamtbild; über einige abwesende Emeriti hörte man, sie seien deswegen bewusst und verärgert weggeblieben; andere Pensionäre fanden die Entscheidung gerade gut. Dennoch oder gerade deswegen kamen rund 400 Teilnehmer, eine deutliche Steigerung gegenüber Passau mit 250.

Zum Programm der Verjüngung passte es auch, dass noch vor der eigentlichen Eröffnung ein „Forum der Jungen“ neu eingerichtet wurde. So etwas praktiziert etwa der Historikertag seit Längerem, und die Münsteraner Kollegen gaben nun auch vier

1 http://www.jura.uni-passau.de/rechtshistoriker/ (zuletzt besucht am 21.10.2010)

2 Miloš Vec, Wie die Alten sungen. Neue Begriffe dringend gesucht: Die Verjüngung der Disziplin fiel auf dem siebenunddreißigsten Deutschen Rechtshistorikertag in Passau aus, in: Frankfurter Allgemeine Zeitung, vom 18. September 2008, S.37. 
Nachwuchsforschern die Gelegenheit, zu reden. Philipp Scheibelreiter (Wien), Anja Steiner (Erlangen), Stephan Dusil (Toronto/Zürich) und David von Mayenburg (Bonn) stellten sich mit ihren laufenden Habilitationsvorhaben vor; sämtlich bereits weit gediehen und professionell präsentiert. Der runde Saal im Schloss war vielleicht nur zu einem Drittel gefüllt. Vielleicht mag auch Unsicherheit im Hinblick auf die Adressaten zu diesen Lücken beigetragen haben: Für wen wurde das „Forum der Jungen “ eigentlich eingerichtet, direkt gefragt: Waren die Nicht-Jungen unerwünscht? Keineswegs, seitens der Veranstalter hieß es ausdrücklich, dass alle „Interessierten herzlich eingeladen sind“, und man freue „sich besonders auch über eine Teilnahme erfahrener Kollegen ". ${ }^{3}$ In der Münsteraner Praxis waren sie sogar die eigentlichen Adressaten. Dazu beigetragen haben mag, dass es das „Annual Forum of Young Legal Historians" gibt, ${ }^{4}$ wo Nachwuchs exklusiv zu Nachwuchs redet, das nächste Mal übrigens im April 2011 in Maastricht. Tatsächlich entwickelte sich in den Münsteraner Diskussionen ein konsistentes Muster rein asymmetrischer Sprecherpositionen: Aus dem Plenum meldeten sich Lehrstuhlinhaber, die vier Habilitanden standen oben auf dem Podium Rede und Antwort auf kluge Fragen, berechtigte Einwände und ernst gemeintes Lob. Die anderen Jungen saßen dabei und schwiegen.

Danach kam Bewegung in das Schloss, das Gros der Kongressteilnehmer drängte in die Aula, wo nun in dem nunmehr erwartungsvoll gefüllt Saal die drei Eröffnungsredner grüßten, bevor der Gast seinen Festvortrag zu Gehör bringen durfte. Der Bonner Theologe und Alttestamentler Ulrich Berges, verwandte in seinem fesselnden Vortrag spontan die Wendung von der „Denkmöglichkeit der Gerechtigkeit“. Berges“ Thema lautete „Gottes Gerechtigkeit im Alten Testament.“ Er widmete sich zunächst den drei Rechtskorpora aus dem 9.-5. Jahrhundert vor Christus. Berges akzentuierte als ein Wesensmerkmal des israelitischen Rechts, dass die Rechtsnormen durch die Gottesgabe vom utopischen Ort der Wüste her dem König und dem Staat als Rechtsquelle entzogen seien. So werde eine „singuläre Rechtsabhängigkeit“ des Königs im Alten Testament sichtbar, die es sonst nirgends im Alten Orient gegeben habe. Im zweiten Teil seines Vortrags sprach Berges über die Gerechtigkeitsvorstellungen im Alten Testament, besonders von der „rettenden Gerechtigkeit“, die den Schwachen vor dem Starken schützen sollte. Anders als in den Nachbarkulturen seien in Israel die Gerechtigkeitsimpulse nicht nur in den Prologen und Epilogen der Gesetzeskorpora zu finden, sondern in die Gesetzesbestimmungen selbst eingedrungen. Soziale Schutzbestimmungen erhielten eine theologische Begründung und wurden dadurch paränetisch eingeschärft. So wurde z.B. die Bestimmung, ein als Pfand genommener Mantel sei am Abend zurückzugeben,- neben der an die Ratio gerichtete

4 http://aylh.org/upcomingforum/ (zuletzt besucht am 21.10.2010). 
Begründung, er sei doch die einzige Bedeckung für die kalten Nächte - durch die göttliche Ermahnung ergänzt: „Wenn er zu mir schreit, höre ich es, denn ich habe Mitleid“ (Ex 22,26). Gerechtigkeitsimpulse im altorientalischen Recht und im Alten Testament seien dort, so Berges in seinem dritten Teil, wo sie sich in Spannung mit den Rechtssatzungen befunden hätten, zugunsten von letzteren entschieden. Zu den humanisierenden Tendenzen im israelitischen Recht gehörten zum einen das Verbot der lebenslangen Schuldknechtschaft für hebräische Sklaven, zum anderen das Zinsverbot gegenüber Volksgenossen. Die Normen der rettenden Gerechtigkeit würden im Alten Testament entweder schöpfungstheologisch oder mit Blick auf die Befreiung Israels aus dem Sklavenhaus Ägyptens legitimiert, so der Festredner. An einigen Stellen ließen sich im Alten Testament zudem wichtige Entgrenzungen über die Binnenmoral des eigenen Volkes hinaus erkennen. Berges schloss seinen Vortrag elegant mit Verweisen darauf, dass die von ihm zitierten Texte und deren Vorstellungen von einer rettenden Gerechtigkeit auch zum kulturellen Erbe Europas und von daher ebenfalls in die juristische Ausbildung gehörten.

Die vier Hauptvorträge am nächsten und übernächsten Morgen begannen mit Wolfgang Kaisers Bericht aus einem laufenden Forschungsprojekt, das ihn auch im zurückliegenden Sommer beschäftigt hatte: „Das Fragment Berolinense - Römisches Recht und Kanonisches Recht im 9. Jahrhundert in Burgund“. Er begeisterte die Zuhörer dabei durch sein kriminalistisches Gespür bei der Verfolgung von Handschriften und ihren Eigentümlichkeiten und nicht zuletzt durch freie Rede und die eigene Begeisterung, mit der er sich diesen Fragen widmete. Welche Kennzeichen weisen die Überlieferungsträger auf, wer hatte Zugang zu ihnen und was konnte man dadurch über die Verbreitung des römisch-justinianischen Rechts lernen? Kaisers Ausführungen hatten dort ihre überzeugendsten Passagen, wo sie akribisch die Handschriften untersuchten, während das Ziel, die Entstehungsregionen und -zeiten der frühen Beschäftigungen mit römisch-justinianischem Recht zu erforschen, eher auf die Aussprache verlagert wurden.

Den zweiten Hauptvortrag des Vormittags hielt Thomas Duve (Frankfurt am Main): „Kirchliche Rechtsgeschichte im frühneuzeitlichen Hispanoamerika - Bilanz und Perspektiven der Forschung “. Er entwarf ein anregendes Panorama der Perspektiven der kirchengeschichtlichen Erforschung Lateinamerikas. Ausgehend von der Schilderung politischer und geografischer Verhältnisse in der Stadt Mexiko als dem Sitz des Erzbischofs widmete er sich den Rechtsbeziehungen im 16. Jahrhundert. Wie war das Verhältnis des Vizekönigtums zur spanischen Krone und zur indigenen Bevölkerung? Handelte es sich um den Fall eines Legal Transplant, eines gigantischen Rechtstransfers? Als methodisches und forschungspolitisches Anliegen Duves kristallisierte sich die Ergänzung der bisherigen europäischen Sichtweisen heraus, die er auch als zu etatistisch kritisierte. Stattdessen empfahl er neue Perspektiven, nämlich 
erstens den Blick von Hispanoamerika aus, zweitens den Versuch, Normbildungsprozesse „vom Punkt Mexiko aus“ zu verstehen und drittens plädierte er dafür, sich den Themen und Quellen zu widmen, die gerade nicht in die Ordnungsschemata des kirchlichen Rechts passen. Die „normative Ordnung des Religiösen“ habe „notwendig etwas Hybrides“, so Duve.

Lutz Raphaels (Trier) Vortrag „Zwischen Duldung und Bürgerstatus - Die Zugehörigkeitsrechte Fremder in der europäischen Rechts- und Sozialgeschichte der Neuzeit" durchmusterte neuzeitliche Migrationsprozesse und fragte nach der Rolle des Rechts. Raphaels Anliegen war die Verbindung von Rechts- und Sozialgeschichte, wobei er ein Plädoyer für Perspektiven „langer Dauer“ formulierte, die auch der Trierer SFB 600 verfolgt. Migration sei spätestens seit dem 16. Jahrhundert ein „Massenphänomen“ geworden, und Recht habe diese Migration reguliert. Europa sei traditionell ein Auswanderungskontinent und erst seit fünfzig Jahren zum $\mathrm{Zu}$ wanderungskontinent geworden, so daher auch die Regularien. In der innereuropäischen Migration würden Sozial- und Integrationschancen gestaltet, Öffnung und Schließung von Gesellschaften fänden durch die rechtliche Fixierung dieser Chancen statt. Dabei lägen Absicht und Wirkung oft weit auseinander. Im Hinblick auf die rechtliche Dimension befand Raphael, dass „einige wenige Rechtsbegriffe [die Migration] dominieren und strukturieren“. Er nannte ius soli, ius sanguinis und ius domizilii als konkurrierende Rechtsprinzipien, verwies auf die Zäsur der Durchsetzung des bürgerlichen Privatrechts an der Wende vom 18. zum 19. Jahrhundert, die als neue Rechtsgleichheit fixierte Differenzen an Bedeutung habe verlieren lassen, Statusfragen seien einerseits entdramatisiert worden, andererseits habe es auch gegenläufige Bewegungen gegeben, so etwa beim Staatsdienst als der Domäne der Einheimischen. Verfassungsgeschichtlich betonte Raphael, dass der europäische Nationalstaat des 19. und 20. Jahrhunderts die „Vollinklusion“ als Leitbild verfolge; so auch in den politischen Debatten. Die Statusdifferenz zwischen In- und Ausländern werde dramatisiert, und zwar vor allem auf nationaler Ebene, während die Gemeinden vergessen würden, obwohl gerade diese aber teilweise autonom über Statusrechte entscheiden. Dabei lasse sich ein „verwirrendes Chaos lokaler und nationaler Regelungen" feststellen. Zwischen 1880 und 1950 sei die Frage der Staatsbürgerschaft ethnisiert und nationalisiert worden (mit Kulminationspunkten in den beiden Weltkriegen), was man als gemeineuropäisches Phänomen betrachten könne. Bei der Erforschung der Ausländerpolitik müssten die Aspekte der Gesetzgebung, der Diskurse und des Verwaltungshandelns gleichermaßen Berücksichtigung finden, da sie erst zusammen ertragreich für die Forschung seien und ein angemessen komplexes Bild von Chancen und Hindernissen ergäben. Als Strukturmuster langer Dauer benannte Raphael erstens den Primat der Gemeindeebene für Inklusion/ Exklusion, zweitens die Beobachtung, dass Fremdheit mit Armut wächst („Fremdheit hat eine Einkom- 
menslinie“), drittens die Existenz radikaler Ausschließung und viertens die Persistenz von Schattenzonen illegaler Praktiken.

Inga Markovits (Austin/ Texas) arbeitet seit mehreren Jahrzehnten über das DDRRecht und seine Juristen und auch ihr Plenarvortrag „Juristen - böse Christen Sozialisten? Die juristische Fakultät der Humboldt-Universität in den DDR-Jahren“ schöpfte nicht nur aus neuen Quellen, sondern einem weiten Erfahrungsschatz. Ihr Vortragstitel spielte auf ältere Schichten der Juristenkritik an, wird oft Luther zugeschrieben, ist tatsächlich aber älter. Der Brückenschlag zur DDR-Geschichte bestand laut Markovits darin, dass auch die Sozialisten „unbedingt glauben“ wollten, und ihr Interesse liege darin, herauszufinden, was aus der Geisteshaltung der Juristen im Sozialismus wurde. Die Ereignisgeschichte, in die sie diese Erzählung einbettete, ist in den Grundzügen vertraut, aber Markovits Stärke lag in der Anreicherung durch interessante Fundstücke aus gedruckten und ungedruckten Quellen oder aus mündlichen Berichten. Die Stichworte lauteten für die Anfangszeit: Austausch des Personals, Hochschulreformen, Aufbruchsstimmung. In der Praxis freilich dauerte es lange, bis der bürgerliche Habitus verschwand, die als zentral angestrebte Verschmelzung von Ideologie, Wissenschaft und Praxis versandte letztlich sogar. Eine Zäsur stellte bekanntermaßen die Babelsberger Konferenz dar, ein „furchteinflößendes Ereignis“, die die Juristen heftigen Angriffen aussetzte, sie zu Bekenntnissen zum sozialistischen Aufbau aufforderte und ihre Arbeit dem Kriterium der Nützlichkeit unterordnete. Andererseits stellte Markovits fest, dass verblüffenderweise gerade der Stalinismus das Festhalten an bürgerlichem Rechtsdenken erleichterte; die Weiterbenutzung bürgerlicher Begriffe schien durch Stalins eigene Schriften legitimiert, etwa die linguistischen Briefe. Die Rechtswissenschaft wurde Auftragnehmerin von Partei und Staat, die Forschungsprojekte waren Auftragsprodukte, Planung und kollektive Arbeit jene Formen, die das ständige Hineinreden durch andere legitimierten: „Waghalsige Einzelschöpfung war nicht mehr möglich“. Viele Produkte mussten in letzter Minute geändert werden, ihre Datierungen waren wichtig, um sich abzusichern, und Ergebenheitsadressen an die Partei immer erwartet. Die Haltung der Juristen gegenüber dem Marxismus-Leninismus ließ sich aus den offiziellen Schriftstücken für den Forscher kaum erschließen, auch in den Akten seien zumeist die Vorgesetzten die Adressaten gewesen, viele Wendungen daher verschlüsselt. Selten fand sie als Forscherin Anzeichen dafür, „was wirklich los gewesen ist“, bekannte Markovits. Die Beschäftigung der Juristen mit dem Marxismus-Leninismus habe anfangs auf Interesse beruht, sei dann aber als Pflichtübung abgetötet worden. Auch aus Sicht der Partei sei dies enttäuschend gewesen. Man hatte sich dort eine Verschmelzung von Recht und Glauben erhofft, aber die Juristen wussten nicht, wie sie das praktisch leisten sollten. Sie suchten im Sinne einer sozialistischen Heilsgeschichte nach Gesetzmäßigkeiten, aber taten dies „ohne einen Hauch von Empirie“ und letztlich ohne großen Erfolg. Dabei fürchtete man einerseits den Druck der Politik (insofern war 
das Signal von Babelsberg wohl verstanden worden und installierte dauerhaft eine Stimmung der Angst), andererseits ängstigte man sich vor Kritik aus Westdeutschland. So agierte man ständig furchtsam, durfte sich einerseits nicht zu weit theoretisch vorwagen, andererseits bargen auch praktische Fragen Gefahren eigener Art. Kein Wunder, dass schließlich die eigentlichen Reformvorschläge eher aus der Verwaltung selbst kamen statt aus den Reihen der Universitätswissenschaftler. Markovits resümierte, die DDR-Juristen seien „keine Helden“ gewesen, aber „wehe dem Land, das Helden nötig hat" (Brecht). Als „unsichere Kantonisten“ nahm sie sie weder als schmiegsam noch als anpassungsfähig wahr. Sie seien eher besonders „,ideologieuntauglich “ und „ideologienutzlos" gewesen, da zu präzise und permanent zweifelnd. Recht war für die rücksichtslose Durchsetzung von Macht „ein methodisches und gefühlsmäßiges Hindernis“, die Rechtsentwicklung daher ein Beitrag zur Auflösung der DDR. Ohne die Juristen, so Markovits, hätte dieser Prozess länger gedauert. Auf Diskussionsbeiträge replizierend, holte Markovits nochmals theoretisch aus und fasste ihr Credo zusammen: Ein instabiles Rechtssystem sei auch für die Partei besser als keines gewesen, ihr lag am Recht, ohne welches das Land schlecht zu regieren gewesen wäre, Entscheidungen mussten rechtlich getroffen werden und zudem habe eine „Hemmung“ gegenüber dem Unrecht als Handlungsoption bestanden. „Unrechtsstaat" sei kein juristisches Wort, sondern ein Schimpfwort, das sie schon gar nicht in den Mund nehmen wolle. Die DDR sei im Laufe der Geschichte dem Recht immer näher gekommen und „Ordnung verhindert größeres Unrecht“, bekundete Markovits. Diese legitimatorische Pointe freilich stieß manchen Anwesenden, die in Kategorien vergleichender Diktaturforschung dachten, sauer auf. Diese Denkmöglichkeit ging ihnen dann doch zu weit.

Von den vier nachmittäglichen Sektionen erwähnt dieser Tagungsbericht nur zwei, mangels eigenen Besuchs kann hier weder über „Strafrecht, Religion und Staat" noch über „Der Einfluss religiöser Vorstellungen auf die Entwicklung des Erbrechts“ berichtet werden. „Zivilverfahrensrecht in Kirche und Staat" bot eine interessante Mischung aus Vorträgen, in denen sich dogmengeschichtliche Perspektiven gut mit dem Blick auf sozialen Wandel und Ideengeschichte ergänzten. Thomas Rüfner (Trier) behandelte die „Beteiligung Dritter im römischen Zivilprozess“ und verglich vor allem die Übernahmelösung mit der Interventionslösung. Als Fallgruppen von praktischer Relevanz identifizierte er dabei Kauf, Verpfändung und Hypothek. Susanne Lepsius schilderte aus dem spätmittelalterlichen Florenz und Lucca „Klagen gegen Amtsträger: öffentliche Verfahren im zivilprozessualen Gewande“. Sie fand verschiedene institutionelle Ausgestaltungen bei ähnlichen Rahmenbedingungen. Die Verfahren zeichneten sich Lepsius zufolge durch kurze Dauer, präzise Ladungen und niedrige Zugangsschwellen aus: „Ein streit- und prozessfreudiges Milieu“ trete uns hier entgegen, in dem die Professionalisierung des Prozesses weit vorangeschritten war. Andreas Thier (Zürich) sprach über „Zeitdeutung und kanonischer Prozess: 
Zur praesumptio ex tempore“. Diese Vermutung diene als Grundlage, so Thier, um eine Tatsache anzunehmen, die sich aus dem Beweis eines anderen Faktums ergebe. Der Regelbestand der praesumptio sei nicht hoheitlich bestimmt gewesen, sondern ein Produkt der Rechtswissenschaft und vor allem der Praxis, wobei Thier verschiedene Stufen ausmachte und dabei Rückschlüsse auf das Zeitverständnis der jeweiligen Epochen unternahm. Tilman Repgen (Hamburg) widmete sich einer neuzeitlichen Dogmengeschichte: "Qui dicit probare debet - Studien zur Beweis(führungs)last“. Die Klammer im Titel deutete eine Wahrnehmungsverschiebung bei der juristischen Konstruktion des Problems an, die das 19. Jahrhundert vorgenommen hatte. Repgen setzte sich insbesondere mit den Positionen Coccejis, Leysers, Webers und Bethmann-Hollwegs - auf dem schließlich die Beweislasttheorien des 19. und 20. Jahrhunderts aufbauen - auseinander und befand resümierend, die Dogmatik der Beweislast sei „substanziell vorangeschritten“.

Die Sektion „Rechtsgeschichte, Rechtsvergleichung und Rechtsdogmatik: historischkritisches Kommentieren“ eröffnete Sonja Meier (Marburg) mit ihrem Beitrag über „Historisch-kritisches Kommentieren am Beispiel des HKK“. Sie stellte den Zuhörern Fragen und Methoden des Projekts vor, wobei sie die praktischen Beispiele ihrer Kommentierung zu den Schuldner- und Gläubigermehrheiten entnahm und die Entscheidungen für die von den Herausgebern und Autoren gewählten Zugriffe auf das BGB erläuterte. Lord Roger of Earlsferry (London) berichtete über „Arguments from legal history in British Courts". Die Besonderheiten lagen dabei in der Rolle von historischen Argumenten im Rahmen eines nicht-kodifizierten Rechtssystems. Dabei unterstrich er die Gefahren, die in solchen Rückgriffen lägen und illustrierte das praktische Vorgehen mit Beispielen aus Zivil-, Straf- und Verfassungsrecht. Filippo Ranieri (Saarbrücken) sprach über „Rechtsgeschichte und Rechtsvergleichung als Reflektionsebene zu den Entwicklungsspielräumen eines Europäischen Zivilrechts“. Jede historische Betrachtung des Privatrechts sei mit einem rechtstheoretischen Anliegen verbunden, die Frage nach Geltung und Veränderung der Privatrechtsnormen immer enthalten. Anders jedoch im Strafrecht, wo die Normen eine völlig andere Funktion hätten. Ranieri fragte, welches Aufklärungspotenzial uns die Rechtsgeschichte für das Privatrecht in Europa biete und notierte vier Entwicklungstrends: 1 . ein verändertes Verständnis der kodifizierten Norm, 2. Dekodifikation und Renationalisierung der Rechtsquellen, 3. formelle Einheit des Privatrechts als Problem und 4. Globalisierungstendenzen des Privatrechts. Er plädierte dafür, dass die Rechtsgeschichte die Funktion einer juristischen Grundlagenforschung übernehmen solle, da sie tiefer liegende Strukturen erkennen könne. Miloš Vec (Frankfurt/Main) widmete sich in „Flaggschiffe und Stiefkinder. Rechtsgeschichte als Kommentierung historischer Normen und rechtspolitischer Kommentar" dem historisch-kritischen Kommentieren in Straf- und Zivilrecht und verglich die laufenden Projekte. Der HKK sei das Vorzeigeprojekt der deutschen Rechtsgeschichte, ein ob seines Anspruchs auf 
methodische und perspektivische Kohärenz auch im internationalen Vergleich einzigartiges Großvorhaben, das seinen kritischen Anspruch in mehrfacher Hinsicht einlöse. Der historische Anspruch hingegen oszilliere zwischen Dogmen- und Problemgeschichte, wobei die letztere ob der Anlage des Projekts strukturelle Schwächen aufweise.

Dass es schwierig ist, Kongresse als Ganze zu charakterisieren, liegt auf der Hand, da Veranstalter, Teilnehmer und Themen sehr plurale Perspektiven einbringen und nicht über einen Kamm geschert werden sollten. Dennoch sind die in zweijährigem Zyklus stattfindenden Rechtshistorikertage Haltepunkte beim Blick auf das ganze Fach und lassen Stimmungen durchblicken, die man mit der gebotenen Vorsicht fassen kann. Auffällig war in diesem Sinne, dass es wohl keine Grundsatzdebatten über die Funktion und Ausrichtung der Rechtsgeschichte gab. Das spricht einerseits für die Konsolidierung des Fachs im Rahmen der juristischen Curricula, wo noch vor Jahren Kürzungen und Stellenwegfall empfindliche Einbußen mit sich brachten. Nun aber hat sich diese Front beruhigt, was man auch beim Kassensturz in der Mitgliederversammlung hören konnte. Exzellenzinitiative und Drittmittelforschung haben sogar zu einem spürbaren Interesse an juristischer Grundlagenforschung geführt, von dem auch die Rechtsgeschichte profitiert. So wurden diesmal keine Rettungsschirme aufgespannt oder alarmistischen Parolen ausgegeben, stattdessen drang nur der Wunsch der Forscher nach kürzeren Sperrfristen des Bundesverfassungsgerichts an die Öffentlichkeit. ${ }^{5}$ Die Frage, ob man aus „Deutscher Rechtshistorikertag“ das nationale Adjektiv tilgen sollte, wurde erneut abschlägig beschieden. Das war konsequent, denn nicht-deutschsprachige Vorträge und Vortragende waren wie gehabt die Ausnahme. Das Fach spürt hierzulande (noch) keinen Anglisierungsdruck und auch eine Internationalisierung wurde weder institutionell diskutiert noch lässt sie sich inhaltlich an den Fragestellungen ablesen. Letztere bewegen sich überwiegend in zivilrechtsgeschichtlichen Bahnen, während Geschichte des Strafrechts und öffentlichen Rechts weiterhin vernachlässigt werden - auch deswegen, weil diese Forschungsgebiete in Deutschland eine schwache Koppelung mit der Lehre aufweisen. Eine Dynamik, die das aufbrechen oder in Frage stellen könnte, ist nicht absehbar.

Andererseits ist es dennoch erstaunlich, dass die Selbstvergewisserungen ausblieben, die ja eben auch durch andere Fragen motiviert sein können. Denn auch Methodenund Richtungsstreitigkeiten fordern Bekenntnisse oder ihre Abwehr heraus, eröffnen Arenen, in denen um Perspektiven und Zwecke historischer Forschung gerungen wird. Offenbar aber taugten in Münster alte Gegensätze nicht mehr zum Disput und neue sind unter den Akteuren (noch) nicht erkennbar. So wurden keine Methodenstreitigkeiten ausgerufen, keine epistemologischen Wenden gefordert oder grund-

5 Martin Otto, Keine Gnade für Doktoren! Der Rechtshistorikertag zu den Karlsruher Akten, in: Frankfurter Allgemeine Zeitung, vom 19. Oktober 2010, S. 35. 
sätzlich neue Prämissen und Prinzipien als unhintergehbare Logiken der Forschung proklamiert. Das spricht für ein pragmatisches Interesse an guter Forschung gleich welcher Couleur und vielleicht auch für die Abwesenheit radikaler politischer oder forschungspolitischer Überzeugungen, deren Wesen oder Zweck oft die Polarisierung ist. Insofern scheinen hier Rechtsgeschichte und Geschichtswissenschaft, wo sich auf dem Berliner Historikertag zwei Wochen später analoge Beobachtungen machen ließen, ähnlichen Zyklen zu folgen. Fragen der übergreifenden Deutung fehlten in den Diskussionen, Reizworte ließen sich keine ausmachen. Nach dem vergangenen Historikerstreit springt kein Rechtshistorikerstreit in die Bresche publikumswirksamer Kontroversen. Legitimationsfragen werden weder von noch an die Forschenden gestellt. Dass es dem externen Beobachter aus der Tagespresse exklusiv vorbehalten war, mit der „Denkmöglichkeit“ durch Rechtsgeschichte ein solches Motiv ins Spiel zu bringen und dass dies peripher blieb, spricht dafür, vielleicht aber auch für eine aktuelle Aversion gegen Theorie- und Legitimationskontroversen prinzipieller Art. Ob man daraus den Schluss ziehen sollte, dass es dort, wo sich keiner aufgeregt hat, nicht aufregend ist, scheint dann aber doch zu pessimistisch. 